\title{
A Competitive Model of Economic Geography
}

\author{
Bryan Ellickson ${ }^{1}$ and William Zame ${ }^{2}$ \\ 1 UCLA, Los Angeles, CA 90049, USA ellickson@econ.ucla.edu \\ 2 UCLA, Los Angeles, CA 90049, USA zame@econ.ucla.edu
}

\begin{abstract}
Most of the literature argues that competitive analysis has nothing interesting to say about location. This paper argues, to the contrary, that a competitive model can have something interesting to say about location, provided that locations are not identical and transportation costs are not zero. To do this, it constructs a competitive intertemporal general equilibrium model and applies it to a suggestive example of migration.
\end{abstract}

Keywords and Phrases: economic geography

JEL Classification Numbers: D5, R0

\section{Introduction}

How should we model the location of economic activity? Most of the literature has taken the point of view that competitive analysis has nothing to say about location. Krugman [7], for instance, writes "Essentially, to say anything useful or interesting about the location of economic activity in space, it is necessary to get away from the constant-returns, perfect competition approach that still dominates most economic analysis." This view arises in part from a theorem of Starrett [14]: if all locations are identical and transportation costs are zero, then perfect competition (constant returns to scale in production, perfect markets, price-taking agents) must lead economic activity to be uniformly distributed in space.

We think the literature has taken the wrong lesson from Starrett's theorem. Locations are not all the same, and transportation costs are not zero, so the question, in our minds, is not whether a competitive model can have anything interesting to say about a world in which locations are identical and transportation costs are zero, but rather whether a competitive model can have anything interesting to say about a world in which locations are not identical and transportation costs are not zero. Put differently, the question is 
not whether competition can lead to a heterogeneous present from a homogenous past, but whether competition can lead to the kind of heterogeneity we actually see in the present from the kind of heterogeneity we see (or imagine) in the past. No one has tried seriously to address this question.

The purpose of this paper is to construct a competitive model that incorporates differences in location and non-zero transportation costs, and to use this model to explore a suggestive example. Our (tentative) conclusion is that competitive models can have something interesting to say. ${ }^{3}$

From the point of view of Debreu [4], location - like time and state of nature - should be incorporated simply by viewing location as part of the description of a commodity, and transportation should be incorporated simply as a particular kind of production. However, this approach is really not satisfactory. The problem is not with locating and transporting commodities, but rather with locating and transporting people. The perspective of our model is that, as has been true throughout most of history, individuals are only able to work and consume in the same location where they reside, and changing that location requires resources and time, and may be unpleasant or even dangerous. As we show in an example, this perspective is especially useful for thinking about migration.

Three other features of our approach merit comment here. The first is that we build a model with a finite number of locations and a continuum of people. Since the surface of the earth appears continuous and its population is surely finite, these choices might seem quite backward, but we believe they are natural. We posit a finite number of locations because we view locations as neighborhoods, cities, counties, or even countries — but not as tiny plots of land in the inner city. We posit a continuum of agents because it eases the modeling of competition and the treatment of indivisibilities inherent in locational choices. We do not view our continuum model as the limiting case of models with large finite numbers of agents but rather as simply a convenient approximation of a fixed model with a large, but definite and finite, number of agents. ${ }^{4}$ (In fact, we adopt a framework in which the population is described entirely in statistical terms, without explicit reference to the number of agents.) We believe our model is quite consistent with the way data is reported and with computational work that applies Scarf's [12] algorithm to locations; see Arnott and MacKinnon [1] or Richter [10,11] for example.

The second is that we treat all goods as potentially mobile, but view mobility as a matter of degree. Land is perfectly immobile, raw materials and the products of agriculture and manufacture are typically mobile - but

\footnotetext{
${ }^{3}$ We don't suggest that constant returns perfect competition is the whole story. See Fujita, Krugman and Venables [5] for analysis based on increasing returns and monopolistic competition.

${ }^{4}$ As Berliant [2] points out, in the locational context there are logical problems with viewing a continuum model as a limit of large finite models; see also Papageorgiu and Pines [9] and Berliant and ten Raa [3].
} 
the cost of transportation may be different for different goods, and will be determined endogenously at equilibrium.

The third is that we take account of time and uncertainty, and allow for both to enter into agents' decision-making. The role of time is quite obvious in our example, which involves migration; uncertainty plays no role in the present paper, but will be treated in subsequent work.

Following this Introduction, Section 2 presents our locational model, Section 3 states and proves our existence theorem, and Section 4 presents the example.

\section{The Locational Model}

In this Section, we lay out the basics of our model and present a simple example to illustrate the ideas.

\subsection{Sites and Trips}

We take as given a finite set $S$ of sites. We view the description of a site as including time and state of nature as well as physical location. If there is only one site, the geography disappears so we assume henceforth that there are at least two sites.

Each consumer is located by an initial specification of site (see below) and by subsequent choices of site. Because changes of site may require resources, it is convenient to view the changes of site, rather than sites, as the actual objects of choice. We therefore define a trip to be a pair $t=\left(s, s^{\prime}\right)$ where $s, s^{\prime} \in S, s \neq s^{\prime}: s$ is the starting point, $s^{\prime}$ is the ending point. We take as given a set of trips $T \subset S \times S$. A path from site $s$ to site $s^{\prime}$ is a finite collection of trips

$$
t_{1}=\left(s_{0}, s_{1}\right), t_{2}=\left(s_{1}, s_{2}\right) \ldots, t_{n}=\left(s_{n-1}, s_{n}\right)
$$

such that $s_{0}=s$ and $s_{n}=s^{\prime}$. Note that there may be many paths between two given sites - or none.

\subsection{Consumption Goods}

Consumption goods are described, just as Debreu [4] suggests, by physical characteristics, location, date, and state of nature. The last three of these are summarized in the site; we assume that physical characteristics belong to a finite set $L$. Thus, consumption goods are indexed by $S \times L$. We abuse notation and write $S, L$ for the cardinalities of the sets of sites and of physical characteristics, as well as for the sets themselves. (And we frequently say that $L$ consumption goods are available at each site.) Thus the space of consumption bundles is $\mathbb{R}^{S \times L}=\mathbb{R}^{S L}$. For simplicity, we assume that consumption goods are perfectly divisible, although there would be no difficulty in allowing for (some) indivisible goods (with appropriate assumptions on preferences and endowments). 


\subsection{Objects of Choice}

Consumption bundles and trips are objects of choice and are priced, so the choice space and price space are

$$
\mathcal{X}=\mathbb{R}^{S L} \times \mathbb{R}^{T}
$$

Write $\pi_{S L}, \pi_{T}$ for the projections of the choice space $\mathcal{X}$ onto the first factor and second factor (respectively). It is convenient to use functional notation and view choices $x$ and prices $p$ as functions

$$
x, p:(S \times L) \cup T \rightarrow \mathbb{R}
$$

Write $x_{S L}, p_{S L}, x_{T}, p_{T}$ for the restrictions of choices and prices. Write $\delta_{(s, \ell)}, \delta_{t}$ for the consumption bundles representing 1 unit of the consumption good $(s, \ell)$ or 1 unit of the trip $t$, respectively.

\subsection{Production}

We follow McKenzie [8] in describing production in terms of an aggregate production possibility set $Y \subset \mathcal{X}$; an element $y \in Y$ is an aggregate activity vector. Thus we allow for production of consumption goods at a single site, for transport of consumption goods between sites, and for transport of individuals between sites. We make appropriate modifications of standard assumptions:

$\mathbf{P} \mathbf{1} Y$ is a closed convex cone

$\mathbf{P 2}-\mathcal{X}_{+} \subset Y$

P3 $\pi_{S L}(Y) \cap \mathbb{R}_{+}^{S L}=\{0\}$

$\mathbf{P} 4$ if $y=\left(y_{S L}, y_{T}\right) \in Y$ then $y^{\prime}=\left(y_{S L}, y_{T}^{+}\right) \in Y$

The second assumption means that we allow for free disposal in production, the third means that there is no free production of consumption goods (but note that we allow for free production of trips), and the fourth means that trips are not used as inputs to production.

\subsection{Consumers}

A consumer is characterized by a choice set $X \subset \mathcal{X}$, an endowment $e \in$ $\mathbb{R}^{S L} \times\{0\}$, an initial site $s \in S$, and a weak preference relation $\succeq$ on $X$, with associated strict preference relation $\succ$. Note that both consumption bundles and trips enter into preferences, and that consumers are endowed with consumption goods but not with trips.

For our purposes, we insist that the choice of a trip is indivisible and that consumers can choose at most one trip between given sites (keep in mind that sites are dated):

C1 for all $x \in X: x_{T}(T) \subset\{0,1\}$ 
Note that a consumer's choice of trips might also be subject to further restrictions (depending on interpretation).

We insist that consumption can only take place at sites where the consumer is located. To express this idea, say that the site $s^{\prime}$ is accessible from the site $s$ given a choice function $x_{T}$ if there is a path $t_{1}, \ldots, t_{n}$ from $s$ to $s^{\prime}$ such that $x_{T}\left(t_{i}\right)=1$ for each $i$. If $s$ is the initial site then a consumer who chooses $x$ occupies the site $s^{\prime}$ (at some point during the period modeled) exactly when $s^{\prime}$ is accessible from $s$ given $x_{T}$. Thus our requirement is formalized by:

C2 for all $x \in X$ : if $s^{\prime}$ is not accessible from $s$ given $x_{T}$ then $x_{S L}\left(s^{\prime}, \cdot\right) \equiv 0$

Subject to these two restrictions, we assume only that consumption sets are closed subsets of the positive orthant (so that only negative consumption is not permitted):

C3 $X \subset \mathbb{R}_{+}^{S L} \times \mathbb{R}_{+}^{T}$ is a closed set

Preferences satisfy the usual requirements:

C4 the weak preference relation $\succeq$ is reflexive, transitive, complete, and has closed graph in $X \times X$

C5 the strict preference relation $\succ$ is irreflexive, transitive, and has open graph in $X \times X$

C6 preferences are locally non-satiated in consumption goods; that is, for each $x=\left(x_{S L}, x_{T}\right) \in X$ and each $\varepsilon>0$ there is a vector $x_{S L}^{\prime} \in \mathbb{R}^{S L}$ such that $x^{\prime}=\left(x_{S L}^{\prime}, x_{T}\right) \in X,\left\|x_{S L}^{\prime}-x_{S L}\right\|<\varepsilon$, and $x^{\prime} \succ x$

We do not require that the endowment $e$ belongs to the choice set $X$, but we do need to require that the consumer can survive without trade. To this end, say that $x \in X$ is a survival choice if $x-e \in Y$. We require

C7 there exists at least one survival choice

Note that, if production plans make non-positive profits (which will necessarily be the case at equilibrium), then a survival choice will be in the consumer's budget set with respect to every price system.

Finally, we require that the consumer will never make a choice which involves no consumption goods:

C8 if $x \in X$ and $x_{S L} \equiv 0$ then there is a survival choice $x^{\prime}$ such that $x^{\prime} \succ x$

\subsection{The Economy}

For our purposes, it is convenient to view the consumer sector of the economy entirely in statistical terms, as suggested by Hart, Hildenbrand and Kohlberg $[6] .^{5}$ That is, a consumer sector is a probability measure $\mu$ on the space $\mathcal{C}$ of consumer characteristics. (We give $\mathcal{C}$ the topology of closed convergence,

\footnotetext{
${ }^{5}$ We emphasize that this modeling choice is a convenience, rather than a requirement; we could easily formulate the model and establish existence in a framework
} 
viewing the components of the 4-tuple $(X, e, s, \succeq)$ of consumer characteristics as closed subsets of $\mathcal{X}, \mathbb{R}^{S L}, S, \mathcal{X} \times \mathcal{X}$, respectively.) As usual, the interpretation we have in mind is that, for any set $Q$ of consumer characteristics, $\mu(Q)$ represents the fraction of the total consumer population whose characteristics lie in $Q$

An economy is a pair $\mathcal{E}=\langle\mu, Y\rangle$ consisting of a consumer sector and an aggregate production set.

We assume throughout that all goods are potentially available in the aggregate

$\mathbf{E} 1\left(Y+\int e d \mu\right) \cap\left(\mathbf{R}_{++}^{S L} \times \mathbf{R}_{++}^{T}\right) \neq \emptyset$

\subsection{Equilibrium}

An equilibrium for the economy $\mathcal{E}=\langle\mu, Y\rangle$ consists of a price vector $p \in\left(\mathbb{R}_{+}^{S L} \times \mathbb{R}_{+}^{T}\right) \backslash\{0\}$, a probability measure $\nu$ on $\mathcal{C} \times \mathcal{X}$, and a production vector $y \in Y$ such that

(1) the marginal of $\nu$ on $\mathcal{C}$ is $\mu$

(2) $\int(x-e) d \nu=y$ (markets clear)

(3) $\nu(\{(X, e, s, \succeq, x): x \notin X$ or $p \cdot x>p \cdot e\})=0$ (almost all consumers choose in their budget set)

(4) $\nu\left(\left\{(X, e, s, \succeq, x): \exists x^{\prime} \in X, p \cdot x^{\prime} \leq p \cdot e \& x^{\prime} \succ x\right\}\right)=0$ (almost all consumers optimize)

(5) $p \cdot y=\max \left\{p \cdot y^{\prime}: y^{\prime} \in Y\right\}=0$

(production profits are maximized)

A quasi-equilibrium differs from an equilibrium in that only the following weaker version of (4) is satisfied:

$\left(4^{\prime}\right) \nu\left(\left\{(X, e, s, \succeq, x): \exists x^{\prime} \in X, p \cdot x^{\prime}<p \cdot e \& x \succ x^{\prime}\right\}\right)=0$

(almost all consumers quasi-optimize)

We caution the reader that some commodity and trip prices may be 0 at equilibrium. (The price of a commodity may be 0 if no one chooses to locate at the site at which that commodity is available.) However, as the following simple proposition shows, not all commodity prices can be 0 at equilibrium (or quasi-equilibrium).

Proposition If $(p, \nu, y)$ is a quasi-equilibrium for the economy $\mathcal{E}$ then the price of some consumption good is strictly positive.

with a non-atomic measure space of agents. Indeed, we could easily formulate the model in a framework with a finite number of agents, although the indivisible nature of choices and the non-convexity of consumption sets and preferences would mean that equilibrium need not exist. 
Proof Suppose not. By definition, not all prices are zero, so the price of some trip $t^{*}$ must be strictly positive. Assumption $\mathbf{E} 1$ guarantees that $t^{*}$ is produced, and Assumption $\mathbf{P} 4$ guarantees that $t^{*}$ can be produced from consumption goods alone. By supposition, these consumption goods have price zero, so $t^{*}$ can be produced at a positive profit. Since $Y$ is a cone, this means that arbitrarily large production profits are possible, and hence that no production plan can maximize profit; this contradicts the definition of quasi-equilibrium.

\section{Existence of Equilibrium}

Our existence result is the following:

Theorem Every economy $\mathcal{E}=\langle\mu, Y\rangle$ satisfying the assumptions $\mathbf{P 1}$ - P4, C1 - C8, E1 above admits a quasi-equilibrium.

Before beginning the proof, we record some notation and a useful lemma. If $E, F$ are topological spaces, we write $M(E), M(F)$ for the spaces of (regular Borel) measures on $E, F$. If $f: E \rightarrow F$ is a continuous function and $\mu \in M(E)$ is a regular Borel measure on $E$, we write $f_{*}(\mu)$ for the direct image measure on $F$, whose value on a Borel set $G \subset F$ is

$$
f_{*}(\mu)(G)=\mu\left(f^{-1}(G)\right)
$$

Notice that if $E=F \times F^{\prime}$ is a product and $f$ is the projection on the first factor, then $f_{*}(\mu)$ is the marginal of $\mu$ on $\mathrm{F}$.

Lemma Let $A$ be a separable metric space, let $B$ be a compact metric space and let $\pi: A \times B \rightarrow A$ be the projection on the first factor. Let $C \subset A \times B$ be a closed set such that $\pi(C)=A$. If $\mu$ is a positive measure on $A$ then

$$
\Gamma=\left\{\nu \in M(C): \pi_{*}(\nu)=\mu\right\}
$$

is a non-empty, weak-star compact convex subset of $M(C)$.

Proof Convexity of $\Gamma$ is trivial, and compactness is straightforward. To see that $\Gamma$ is not empty, suppose first that $\mu$ is compactly supported, with support $\operatorname{supp} \mu$. Choose a sequence $\left\{\mu_{n}\right\}$ of atomic measures, each of which has support a finite subset of $\operatorname{supp} \mu$, such that $\mu_{n} \rightarrow \mu$ weak star. Since $B$ is compact so is $A^{*}=\pi^{-1}(\operatorname{supp} \mu) \cap C$, and $\pi\left(A^{*}\right) \supset \operatorname{supp} \mu$. For each $n$, write $\mu_{n}=\sum_{k} a_{n}^{k} \delta_{x_{n}^{k}}$, choose points $c_{n}^{k} \in A^{*}$ with $\pi\left(c_{n}^{k}\right)=x_{n}^{k}$, and set $\nu_{n}=\sum_{k} a_{n}^{k} \delta_{c_{n}^{k}}$. It is evident that $\pi_{*}\left(\nu_{n}\right)=\mu_{n}$ and that any weak star limit point $\nu$ of the sequence $\left\{\nu_{n}\right\}$ is a measure supported on $A^{*} \subset C$ for which $\pi_{*}(\nu)=\mu$.

Now consider an arbitrary measure $\mu$. Since $A$ is a separable metric space, every measure on $A$ is tight. Hence we can choose a sequence $\left\{K^{i}\right\}$ of disjoint compact subsets of $A$ such that $\mu\left(\bigcup K^{i}\right)=\mu(A)$. Setting $\mu^{i}=\mu \mid K^{i}$ 
and applying the result in the compactly supported case yields measures $\nu^{i}$ supported on $C \cap \pi^{-1}\left(K^{i}\right)$ such that $\pi_{*}\left(\nu^{i}\right)=\mu^{i}$. Setting $\nu=\sum_{i} \nu^{i}$ yields a measure $\nu$ on $C$ such that $\pi_{*}(\nu)=\mu$, as desired.

We now turn to the proof of the Theorem.

Proof of Theorem Let $\mathcal{E}=\langle\mu, Y\rangle$ be an economy satisfying the assumptions. We construct perturbed economies $\mathcal{E}_{n}=\left(\mu_{n}, Y_{n}\right)$, find equilibria for these perturbed economies, and then obtain a quasi-equilibrium for $\mathcal{E}$ by a limiting argument.

Step 1 We first construct the perturbed economies $\mathcal{E}_{n}$. To construct the consumption sector $\mu_{n}$, first define $\tau^{n}: \mathcal{C} \rightarrow \mathcal{C}$ by

$$
\tau^{n}(X, e, s, \succeq)=\left(X, \frac{1}{n} \mathbf{1}+\inf \left\{e+\frac{1}{n} \mathbf{1}, n \mathbf{1}\right\}, s, \succeq\right)
$$

Set $\mu_{n}=\tau_{*}^{n}(\mu)$. To construct the production sector $Y_{n}$, first note that, by assumption, $Y$ is a closed convex cone and $\pi_{S L}(Y) \cap \mathbb{R}_{+}^{S L}=\{0\}$ (no free production of consumption goods). Hence we may we can find $d>0$ such that $\left\|\pi_{S L}(y)^{-}\right\| \geq \delta\left\|\pi_{S L}(y)^{+}\right\|$for each $y \in Y$. (That is, the marginal rate of transformation for consumption goods is bounded.) Define $Y_{n}$ by

$$
Y_{n}=\left\{y \in \mathbf{R}^{S L} \times \mathbb{R}^{T}: \exists y^{\prime} \in Y, y_{T}^{\prime}=y_{T},\left\|y-y^{\prime}\right\| \leq \frac{d}{2 n}\left\|y^{-}\right\|\right\}
$$

It is easily checked that $\mathcal{E}_{n}$ satisfies the assumptions P1 - P4, C1 - C8, E1.

Step 2 We construct a compact price simplex for $\mathcal{E}_{n}$. Define $Y_{n}^{\circ}$ to be the polar cone of $Y_{n}$; that is

$$
Y_{n}^{\circ}=\left\{p \in \mathbb{R}^{S L} \times \mathbb{R}^{T}: p \cdot y \leq 0 \text { for all } y \in Y_{n}\right\}
$$

Because $Y_{n} \supset-\left(\mathbf{R}_{+}^{S L} \times \mathbf{R}_{+}^{T}\right)$, it follows that $Y_{n}^{\circ} \subset \mathbf{R}_{+}^{S L} \times \mathbf{R}_{+}^{T}$. Define a normalized price simplex by

$$
\Delta_{n}=\left\{p \in Y_{n}^{\circ}: \sum_{(s, \ell) \in S \times L} p(s, \ell)+\sum_{t \in T} p(t)=1\right\}
$$

Note that $\Delta_{n}$ is a compact convex subset of $\mathbf{I R}_{+}^{S L} \times \mathbf{I R}_{+}^{T}$.

We claim that, for $p \in \Delta_{n}$, prices of consumption goods are bounded away from 0: that is, there is some $\varepsilon>0$ such that $p(s, \ell) \geq \varepsilon$ for each $p \in \Delta_{n}$. To see this, suppose first that $p \in \Delta_{n}$ and $p_{S L} \equiv 0$. Our normalization guarantees that $p(t)>0$ for some trip $t$. Assumption E1 guarantees that $t$ is produced, and Assumption $\mathbf{P} 4$ guarantees that $t$ can be produced from consumption goods: there is a $y \in Y_{n}$ such that $\pi_{T}\left(y^{-}\right)=0$ and $y^{+}(t)>0$. Since the prices of consumption goods are identically 0 and the prices of trips are non-negative, it follows that $p \cdot y>0$, which contradicts the fact that $p \in Y_{n}^{\circ}$. We conclude that there is no $p \in \Delta_{n}$ for which $p_{S L} \equiv 0$. 
Because $\Delta_{n}$ is compact, it follows immediately that there is an $\varepsilon_{1}>0$ such that $\sum_{(s, \ell)} p(s, \ell) \geq \varepsilon_{1}>0$ for each $p \in \Delta_{n}$. Set $\varepsilon=\frac{\varepsilon_{1}}{S L} \frac{d}{2 n}$. Fix $p \in \Delta_{n}$ and $\left(s^{*}, \ell^{*}\right) \in S \times L$. Because $\sum p(s, \ell) \geq \varepsilon_{1}$, there is at least one $\left(s^{\prime}, \ell^{\prime}\right)$ for which $p\left(s^{\prime}, \ell^{\prime}\right) \geq \frac{\varepsilon_{1}}{S L}$. By Assumption P2, $-\delta_{\left(s, \ell^{*}\right)} \in Y$; by construction, $\left(\frac{d}{2 n} \delta_{\left(s^{\prime}, \ell^{\prime}\right)}-\delta_{(s *, \ell *)}\right) \in Y_{n}$. Because $p \in Y_{n}^{\circ}$, it follows that

$$
\frac{d}{2 n} p\left(s^{\prime}, \ell^{\prime}\right)-p\left(s^{*}, \ell *\right)=p \cdot\left(\frac{d}{2 n} \delta_{\left(s^{\prime}, \ell^{\prime}\right)}-\delta\left(s^{*}, \ell^{*}\right)\right) \leq 0
$$

whence

as required.

$$
p\left(s^{*}, \ell^{*}\right) \geq \frac{\varepsilon_{1}}{S L} \frac{d}{2 n}=\varepsilon
$$

Step 3 Now we construct an equilibrium for $\mathcal{E}_{n}$ as a fixed point of an excess demand correspondence. By construction, endowments in the support of $\mu_{n}$ are bounded. Since the consumption components of prices in $\Delta_{n}$ are bounded away from 0 , we can find a compact set $B \subset \mathcal{X}$ that contains all the budgetfeasible choices for a consumer whose characteristics lie in $\operatorname{supp} \mu_{n}$; that is: if $p \cdot x \leq p \cdot e$ for some $(X, e, s, \succeq) \in \operatorname{supp} \mu_{n}$ then $x \in B$. For $p \in \Delta_{n}$, set

$$
C(p)=\left\{(X, e, s, \succeq, x) \in \mathcal{C} \times B: p \cdot x \leq p \cdot e, \nexists x^{\prime} \in X, p \cdot x^{\prime} \leq p \cdot e, x^{\prime} \succ x\right\}
$$

Note that $(X, e, s, \succeq, x) \in C(p)$ exactly when $x$ is an optimal choice in the budget set of a consumer with characteristics $(X, e, s, \succeq)$. Apply the above Lemma with $A=\mathcal{C}$ (the space of consumer characteristics), $B=B, C=C(p)$ to conclude that the set of measures

$$
\Gamma(p)=\left\{\nu \in M(C(p)): \pi_{*}(\nu)=\mu\right\}
$$

is compact, convex, and non-empty. It is easily checked (using compactness of $B$ ) that the correspondence $p \mapsto \Gamma(p)$ is upper hemi-continuous. ${ }^{6}$

Set $\widehat{B}=B-B=\left\{b-b^{\prime}: b, b^{\prime} \in B\right\}$, and define correspondences

$$
Z: \Delta_{n} \rightarrow \widehat{B} ; \quad V: \widehat{B} \rightarrow \Delta_{n} ; \Phi: \Delta_{n} \times \widehat{B} \rightarrow Z \times \widehat{B}
$$

by

$$
\begin{aligned}
Z(p) & =\left\{\int(x-e) d \nu: \nu \in \Gamma(p)\right\} \\
V(z) & =\arg \max \left\{p \cdot z: p \in \Delta_{n}\right\} \\
\Phi(p, z) & =Z(p) \times V(z)
\end{aligned}
$$

${ }^{6}$ The argument, which we leave to the reader, is entirely standard with the exception of two points. 1) Because endowments do not belong to consumption sets, they do not belong to budget sets either. However, Assumption C7 guarantees that budget sets are always non-empty. 2) Because the choice of trips is indivisible, the budget correspondence of a consumer is not continuous in prices. However, because of Assumption C8, the individually rational part of the budget correspondence is continuous, whence the optimal choice correspondence is upper hemi-continuous. 
Note that $Z(p)$ is the set of aggregate excess demands at the price $p$. The reader will easily verify (using continuity and linearity of integration, and the properties of the correspondence $\Gamma$ ) that $Z, V$ and $\Phi$ are upper hemicontinuous and have non-empty compact convex values. ${ }^{7}$

Because $\Delta_{n} \times \widehat{B}$ is compact and convex and $\Phi$ is an upper hemi-continuous correspondence with non-empty, compact convex values, $\Phi$ has a fixed point $\left(p_{n}, z_{n}\right)$. By definition, $z_{n}=\int(x-e) d \nu_{n}$ for some $\nu_{n} \in \Gamma(p)$.

We claim that $\left(p_{n}, \nu_{n}, z_{n}\right)$ constitute an equilibrium for $\mathcal{E}_{n}$. To see this, we need only show that $z_{n} \in Y_{n}$, so suppose not. Then there is a price $p$ such that $p \cdot z_{n}>\sup \left\{p_{n} \cdot y: y \in Y_{n}\right\}$. Because $0 \in Y_{n}$, it follows that $p \cdot z_{n}>0$. Because $Y_{n}$ is a cone, it follows that $p \cdot y \leq 0$ for each $y \in Y_{n}$ and hence that $p \in Y_{0}^{\circ}$. Normalizing if necessary, we may assume that $p \in Y_{n}^{\circ}$. On the other hand, an optimizing consumer whose preferences are locally non-satiated spends all his income. Integrating across consumers implies Walras's Law in the aggregate: $p_{n} \cdot z_{n}=0$. Combining these facts and recalling that $\left(p_{n}, z_{n}\right)$ is a fixed point of $\Phi$, we obtain

$$
p \cdot z_{n}>0=p_{n} \cdot z_{n}=\sup \left\{p^{\prime} \cdot z_{n}: p^{\prime} \in \Delta_{n}\right\}
$$

This is a contradiction, so we conclude that $z_{n} \in Y_{n}$, and hence that $\left(p_{n}, \nu_{n}, z_{n}\right)$ constitute an equilibrium for $\mathcal{E}_{n}$.

Step 4 To construct a quasi-equilibrium for $\mathcal{E}$ as a limit of the quasi-equilibria just constructed for the perturbed economies $\mathcal{E}_{n}$, our first task is to show that the sequence $\left\{\nu_{n}^{*}\right\}$ of equilibrium measures is tight.

To this end, set $\bar{e}=\int e d \mu$. Because $\pi_{S L}\left(Y_{1}\right) \cap \mathbf{R}^{S L}=\{0\}$, we may choose a $\bar{y} \in Y_{1}$ such that if $y \in Y_{1}$ and $\pi_{S L}(y)^{-} \leq \bar{e}$ then $\pi_{S L}(y)^{+} \leq \pi_{S L}(\bar{y})^{+}$.

For each positive integer $m$ and each $(s, l) \in S \times L$, set

$$
H_{m}(s, l)=\{(X, e, s, \succ, x) \in \mathcal{C} \times \mathcal{X}: x(s, l)>m\}
$$

Note that

$$
\int_{H_{m}(s, l)} x(s, l) d \nu_{n}^{*}>m \nu_{n}^{*}\left(H_{m}(s, l)\right)
$$

On the other hand, since $\nu_{n}^{*}$ is an equilibrium measure,

$$
\int(x-e) d \nu_{n}^{*}=y_{n} \in Y_{n}
$$

Our construction guarantees that

$$
\int e d \nu_{n}^{*}=\int e d \mu_{n} \rightarrow \int e d \mu
$$

Since consumption is non-negative and $Y_{n} \subset Y_{1}$ for each $n$, our construction also guarantees that

\footnotetext{
7 The arguments follow familiar lines, with the exceptions noted in the previous footnote.
} 


$$
\int x d \nu_{n}^{*}=\int e d \nu_{n}^{*}+y_{n} \leq \bar{e}+\bar{y}^{+}
$$

Combining the equations and inequalities (1)-(4)yields

$$
\nu_{n}^{*}\left(H_{m}(s, l)\right) \leq \frac{1}{m}\left[\bar{e}(s, l)+\bar{y}^{+}(s, l)\right]
$$

Now let $\epsilon>0$. Since the single measure $\mu$ is tight, we may choose a compact subset $K \subset \mathcal{C}$ such that $\mu(\mathcal{C} \backslash K)<\epsilon / 2$. Set $H_{m}=\bigcup_{(s, l)} H_{m}(s, l)$. In view of the inequality (5), if $m$ is sufficiently large, then $\nu_{n}^{*}\left(H_{m}\right)<\epsilon / 2$. Setting

$$
K^{*}=\left\{(X, e, s, \succeq, x) \in \mathcal{C} \times \mathcal{X}: e \in K, x \notin H_{m}\right\}
$$

we obtain a compact subset of $\mathcal{C} \times \mathcal{X}$ such that $\nu_{n}^{*}\left(\mathcal{C} \times \mathcal{X} \backslash K^{*}\right)<\epsilon$ for each $n$. Hence the sequence $\left\{\nu_{n}^{*}\right\}$ of equilibrium measures is tight.

Step 5 We now obtain a quasi-equilibrium for $\mathcal{E}$. Note that all the equilibrium prices $p_{n}$ lie in the simplex

$$
\Delta=\left\{p \in-Y^{\circ}: \sum y(s, l)=1\right\}
$$

which is a compact subset of the non-negative orthant $\mathcal{X}_{+}$. Hence some subsequence of the price sequence $\left(p_{n}\right)$ converges. A tight sequence of measures contains a convergent subsequence. Passing to an appropriate subsequence, therefore, we may assume there is a price $p \in \Delta$ and a measure $\nu$ on $\mathcal{X} \times \mathrm{X}$ such that $p_{n} \rightarrow p$ and $\nu_{n} \rightarrow \nu$. Weak star convergence implies that

$$
y_{n}=\int(x-e) d \nu_{n} \rightarrow \int(x-e) d \nu=y
$$

Because $y_{n} \in Y_{n}$ and each $Y_{n}$ is closed, it follows that $y \in Y_{n}$ for each $n$ and hence that $y \in Y=\bigcap_{n} Y_{n}$. It is now easily verified that $(\nu, p, y)$ constitutes a quasi-equilibrium for the economy $\mathcal{E}$.

Note that we have only established the existence of a quasi-equilibrium, not of an equilibrium. Because trips are indivisible and agents can only consume at sites they occupy, it is not hard to find simple examples of quasi-equilibria that are not equilibria; indeed, it is not hard to find economies that admit no equilibrium at all. It seems difficult to write down simple and economically natural assumptions sufficient to guarantee that every quasi-equilibrium is an equilibrium, or even that an equilibrium exists - although the example below does enjoy these properties.

\section{Example}

In this Section we present an example that addresses migration in a way rather different from the literature (compare Sjaastaad [13] for instance), and 
demonstrates our thesis that a small inhomogeneity in locations can lead to a large and striking inhomogeneity at equilibrium: substantial migration flows, both forward and backward.

There are 2 locations (East and West) and 3 dates $(0,1,2)$, but all consumers are located in the East at date 0 , so we identify 5 sites $E 0, E 1, W 1$, $E 2, W 2$ and 6 trips:

$$
\begin{aligned}
t_{1}=(E 0, E 1), t_{2} & =(E 0, W 1) \\
t_{3}=(E 1, E 2), t_{4} & =(E 1, W 2) \\
t_{5}=(W 1, W 2), t_{6} & =(W 1, E 2)
\end{aligned}
$$

That is, consumers, who are located in the East at date 0, can remain in the East at date 1 or migrate to the West at date 1 ; consumers who remain in the East at date 1 can remain in the East at date 2 or migrate to the West at date 2 ; consumers who have moved to the West at date 1 can remain in the West at date 2 or return to the East at date 2. (Migration occurs between dates.) The possible choices of paths and consequent choices of location through time are:

$$
\begin{aligned}
& \pi_{1}=\left(t_{1}, t_{3}\right): \text { East at dates } 0,1,2 \\
& \pi_{2}=\left(t_{1}, t_{4}\right): \text { East at dates } 0,1 \text {; West at date } 2 \\
& \pi_{3}=\left(t_{2}, t_{5}\right): \text { East at date } 0, \text { West at dates } 1,2 \\
& \pi_{4}=\left(t_{2}, t_{6}\right): \text { East at date } 0, \text { West at date } 1, \text { East at date } 2
\end{aligned}
$$

There are 2 commodities at each site, land and corn; we write $E 0 c, E 0 \ell$ for corn, land at site $E 0$, and so forth. Agents are endowed with (claims to) $k$ acres of land at each site, but no corn. Corn can be produced using land as the sole input: 1 acre of land yields 1 ton of corn at the same date and location. Corn is perishable, and cannot be transported from one site to another. Each trip between distinct sites uses as input $\tau$ tons of corn at the initial site.

Agents derive utility only from the consumption of corn, according to the utility function

$U(x)=\ln x(E 0 c)+\beta[\ln x(E 1 c)+\alpha \ln x(W 1 c)]+\beta^{2}[\ln x(E 2 c)+\alpha \ln x(W 2 c)]$

(Recall that $x(E 0 c)$ represents corn consumed in the East at date 0 , and so forth). The parameter $\beta(0<\beta \leq 1)$ represents time discounting, the parameter $\alpha>0$ represents the weighting of West versus East: if $\alpha<1$, agents prefer the East (other things equal); if $\alpha>1$ agents prefer the West. To solve for equilibrium, let $\rho_{j}(j=1,2,3,4)$ be the fraction of agents who choose the path $\pi_{j}$.

Because agents derive no utility from consuming land directly, all land will be used for corn production. Moreover, zero profit in corn production implies that the price of 1 ton of corn equals the price of 1 acre of land, so we henceforth suppress land prices entirely. Write

$$
p=(p(E 0 c), p(E 1 c), p(E 2 c), p(W 1 c), p(W 2 c))
$$


for the vector of corn prices, and normalize so that $p(E 0 c)=1$. Given these prices for corn and the implied prices for land, all agents hold wealth:

$$
w(p)=[1+p(E 1 c)+p(E 2 c)+p(W 1 c)+p(W 2 c)] k
$$

Write $w_{j}(p)$ for the net wealth of an agent who chooses path $\pi_{j}$.

If $\tau$ is small enough then at equilibrium all sites will be occupied, whence all corn will be consumed. Hence market clearing at each site yields:

- $\quad$ Site $E 0$

$$
\begin{aligned}
\rho_{1} \frac{w_{1}(p)}{1+\beta+\beta^{2}}+ & \rho_{2} \frac{w_{2}(p)}{1+\beta+\beta^{2} \alpha}+\rho_{3} \frac{w_{3}(p)}{1+\beta \alpha+\beta^{2} \alpha} \\
& +\quad \rho_{4} \frac{w_{4}(p)}{1+\beta \alpha+\beta^{2}}+\rho_{3} \tau+\rho_{4} \tau=k
\end{aligned}
$$

- Site E1:

$$
\rho_{1} \frac{\beta w_{1}(p)}{\left(1+\beta+\beta^{2}\right) p(E 1 c)}+\rho_{2} \frac{\beta w_{2}(p)}{\left(1+\beta+\beta^{2} \alpha\right) p(E 1 c)}+\rho_{2} \tau=k
$$

- Site E2:

$$
\rho_{1} \frac{\beta^{2} w_{1}(p)}{\left(1+\beta+\beta^{2}\right) p(E 2 c)}+\rho_{4} \frac{\beta^{2} w_{4}(p)}{\left(1+\beta \alpha+\beta^{2}\right) p(E 2 c)}=k
$$

- Site $W 1$ :

$$
\rho_{3} \frac{\beta \alpha w_{3}(p)}{\left(1+\beta \alpha+\beta^{2} \alpha\right) p(W 1 c)}+\rho_{4} \frac{\beta \alpha w_{4}(p)}{\left(1+\beta \alpha+\beta^{2}\right) p(W 1 c)}+\rho_{4} \tau=k
$$

- Site $W 2$ :

$$
\rho_{2} \frac{\beta^{2} \alpha w_{2}(p)}{\left(1+\beta+\beta^{2} \alpha\right) p(W 2 c)}+\rho_{3} \frac{\beta^{2} \alpha w_{3}(p)}{\left(1+\beta \alpha+\beta^{2} \alpha\right) p(W 2 c)}=k
$$

Because consumers have identical preferences and endowments, they must attain equal levels of utility in equilibrium. Direct calculation yields indirect utility functions $v_{j}(p)$ conditional on trip chosen, expressed as functions of corn prices:

$$
\begin{aligned}
& v_{1}(p)=\ln (\left.\frac{w_{1}(p)}{1+\beta+\beta^{2}}\right)+\beta \ln \left(\frac{\beta w_{1}(p)}{\left(1+\beta+\beta^{2}\right) p(E 1 c)}\right) \\
&+\beta^{2} \ln \left(\frac{\beta^{2} w_{1}(p)}{\left(1+\beta+\beta^{2}\right) p(E 2 c)}\right) \\
& v_{2}(p)=\ln \left(\frac{w_{2}(p)}{1+\beta+\beta^{2} \alpha}\right)+\beta \ln \left(\frac{\beta w_{2}(p)}{\left(1+\beta+\beta^{2} \alpha\right) p(E 1 c)}\right) \\
&+\beta^{2} \alpha \ln \left(\frac{\beta^{2} \alpha w_{2}(p)}{\left(1+\beta+\beta^{2} \alpha\right) p(W 2 c)}\right)
\end{aligned}
$$




$$
\begin{gathered}
v_{3}(p)=\ln \left(\frac{w_{3}(p)}{1+\beta \alpha+\beta^{2} \alpha}\right)+\beta \alpha \ln \left(\frac{\beta \alpha w_{3}(p)}{\left(1+\beta \alpha+\beta^{2} \alpha\right) p(W 1 c)}\right) \\
+\beta^{2} \alpha \ln \left(\frac{\beta^{2} \alpha w_{3}(p)}{\left(1+\beta \alpha+\beta^{2} \alpha\right) p(W 2 c)}\right) \\
v_{4}(p)=\ln \left(\frac{w_{4}(p)}{1+\beta \alpha+\beta^{2}}\right)+\beta \alpha \ln \left(\frac{\beta \alpha w_{4}(p)}{\left(1+\beta \alpha+\beta^{2}\right) p(W 1 c)}\right) \\
+\beta^{2} \ln \left(\frac{\beta^{2} w_{4}(p)}{\left(1+\beta \alpha+\beta^{2}\right) p(E 2 c)}\right)
\end{gathered}
$$

At equilibrium, identical consumers obtain the same utility, so:

$$
v_{1}(p)=v_{2}(p)=v_{3}(p)=v_{4}(p)
$$

Finally, the fractions of consumers choosing each path must add to 1 :

$$
\rho_{1}+\rho_{2}+\rho_{3}+\rho_{4}=1
$$

Boiling down equations (6)-(16) yields 9 equations in 8 unknowns: corn prices in 4 sites (recall the normalization $p(E 0 c)=1$ ) and the 4 fractions $\rho_{j}$. However, Walras' Law means that one of these equations are redundant, leaving 8 independent equations in 8 unknowns. An equilibrium is determined by a solution to these equations having the additional property that each of the fractions $\rho_{j}$ lies between 0 and 1 . Except for special values of the parameters it does not seem possible to solve these equations in closed form, but it is not difficult to solve numerically. To ease the computational burden, we take $\beta=1$, so consumers do not discount the future. We also fix $k=10$, just for purposes of illustration.

First consider the homogeneous case: $\tau=0$ and $\alpha=1$. In this case, half the population moves to the West at date 1, equalizing land rents and corn production in both locations from date 1 on. Migration flow between dates 1 and 2 is indeterminate (consumers do not care about location and travel is costless). This is competition in a world without spatial differentiation or transportation cost, and the outcome is perfectly homogeneous, exactly as Starrett's [14] theorem predicts.

Second consider a small cost of moving: $\tau=0.01$ (this is $0.1 \%$ of the production of each agent's land endowment at each site), but $\alpha=1$ (so that agents remain indifferent between East and West). As shown in the middle rows of Tables $1-4$, the result is essentially the same: ${ }^{8}$ at equilibrium, half the population moves to the West at date 1 , but no one moves thereafter; land and

\footnotetext{
${ }^{8}$ To the precision presented in the Tables, the result is exactly the same, but the small cost of moving does make a difference: for example,
}

$p(E 1 c) \approx 0.5000001 \quad p(E 2 c) \approx 0.499750 \quad p(W 1 c) \approx 0.499500 \quad p(W 2 c) \approx 0.499750$ 
corn prices are equalized at all sites with twice as much land in production, so consumption of corn per capita doubles as well.

Third consider $\tau=0.01, \alpha \neq 1$. As we see in Table 1 , if $\alpha=0.92$ (consumers prefer the East), slightly more than half of all consumers spend their whole lives in the East; the rest migrate West in date 1 and remain there in date 2. Agents who migrate West pay less for land and corn and, as shown in Tables 2-4, consume more corn, compensating them for the undesirability of the West and the cost of migrating. If $\alpha=1.08$ (consumers prefer the West), slightly more than half of all consumers migrate West in date 1 and stay there; all the rest remain in the East throughout. Agents who migrate West pay more for land and corn, and hence consume less corn, but are compensated by the desirability of the West. (The imperfect asymmetry of parameters and outcomes reflects the initial asymmetry that all consumers begin in the East.)

Table 1. Paths and prices $(\beta=1, k=10, \tau=.01)$

\begin{tabular}{ccccccccc}
\hline$\alpha$ & $\rho_{1}$ & $\rho_{2}$ & $\rho_{3}$ & $\rho_{4}$ & $p(E 1 c)$ & $p(E 2 c)$ & $p(W 1 c)$ & $p(W 2 c)$ \\
\hline 0.92 & 0.56 & 0.00 & 0.44 & 0.00 & 0.54 & 0.54 & 0.42 & 0.42 \\
0.94 & 0.54 & 0.00 & 0.46 & 0.00 & 0.53 & 0.53 & 0.44 & 0.44 \\
0.96 & 0.39 & 0.13 & 0.34 & 0.13 & 0.52 & 0.52 & 0.46 & 0.46 \\
0.98 & 0.45 & 0.06 & 0.43 & 0.06 & 0.51 & 0.51 & 0.48 & 0.48 \\
1.00 & 0.50 & 0.00 & 0.50 & 0.00 & 0.50 & 0.50 & 0.50 & 0.50 \\
1.02 & 0.42 & 0.07 & 0.44 & 0.07 & 0.49 & 0.49 & 0.52 & 0.52 \\
1.04 & 0.36 & 0.11 & 0.41 & 0.11 & 0.48 & 0.48 & 0.54 & 0.54 \\
1.06 & 0.12 & 0.34 & 0.20 & 0.34 & 0.47 & 0.47 & 0.56 & 0.56 \\
1.08 & 0.45 & 0.00 & 0.55 & 0.00 & 0.46 & 0.46 & 0.58 & 0.58
\end{tabular}

All of this seems reasonable, if not particularly surprising. However, for values of $\alpha$ closer to 1 , a much more interesting phenomenon occurs: some consumers migrate from East to West in date 1 and then migrate back at date 2, to be replaced by new migrants from the East. As shown in Table 1 and Figure 1, for $\alpha<1$ (the East is preferred), the backward flow of migration reaches a peak $\left(\rho_{2}=\rho_{4}=0.13\right)$ at around $\alpha=0.96$. A similar phenomenon occurs for $\alpha>1$, with the offsetting flows reaching a peak $\left(\rho_{2}=\rho_{4}=0.34\right)$ at around $\alpha=1.06$. (Again, note the imperfect symmetry between these parameter values.) Both of these outcomes reflect a general equilibrium phenomenon: In the first instance, Easterners migrate West to take advantage of low prices/abundant land in the West, but later return to the more attractive East. In the second instance, Easterners migrate to the more attractive West, but return because Western land is expensive.

Reverse migration seems striking - and unexpected, although well-documented in the empirical literature - especially because it might seem to suggest that resources are wasted and that social welfare could be improved by eliminating 


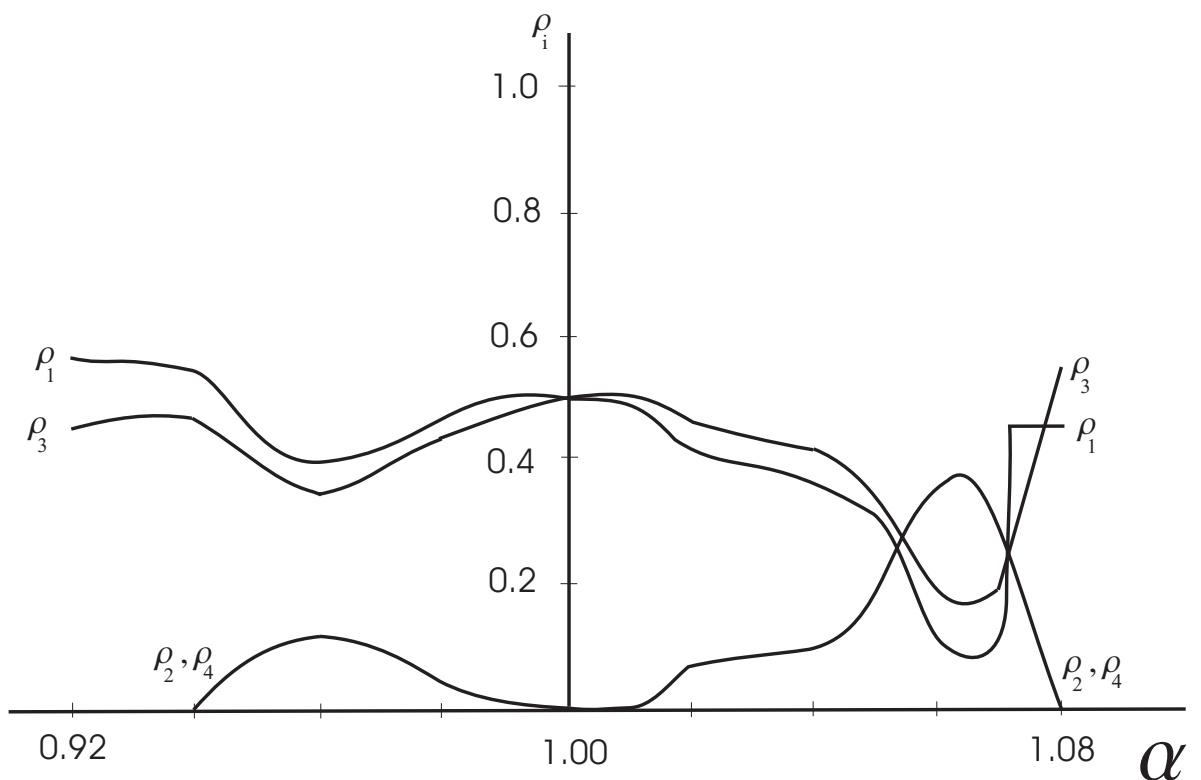

Fig. 1. Path probabilities as functions of $\alpha$

Table 2. Corn consumption, date $0(\beta=1, k=10, \tau=.01)$

\begin{tabular}{ccccc}
\hline$\alpha$ & $x_{1}(E 0) \rho_{1}$ & $x_{2}(E 0)$ & $x_{3}(E 0)$ & $x_{4}(E 0)$ \\
\hline 0.92 & 9.8 & 10.0 & 10.3 & 10.0 \\
0.94 & 9.8 & 10.0 & 10.2 & 10.0 \\
0.96 & 9.9 & 10.0 & 10.1 & 10.0 \\
0.98 & 9.9 & 10.0 & 10.1 & 10.0 \\
1.00 & 10.0 & 10.0 & 10.0 & 10.0 \\
1.02 & 10.1 & 10.0 & 9.9 & 10.0 \\
1.04 & 10.1 & 10.0 & 9.9 & 10.0 \\
1.06 & 10.2 & 10.0 & 9.8 & 10.0 \\
1.08 & 10.3 & 10.0 & 9.8 & 10.0
\end{tabular}

reverse migration and making appropriate transfers. Of course, no resources are in fact wasted; as usual, equilibrium is Pareto optimal.

This example echoes a point made in the Introduction. As Starrett's theorem tells us, perfect competition and perfect homogeneity must lead to a homogenous outcome. As this example demonstrates, however, perfect competition and just a little heterogeneity (in this case, a relatively small preference for one location over the other) can lead to a very heterogeneous outcome indeed. 
Table 3. Corn consumption, date $1(\beta=1, k=10, \tau=.01)$

\begin{tabular}{ccccc}
\hline$\alpha$ & $x_{1}(E 1 c)$ & $x_{2}(E 1 c)$ & $x_{3}(W 1 c)$ & $X_{4}(W 1 c)$ \\
\hline 0.92 & 18.0 & 18.5 & 22.5 & 21.9 \\
0.94 & 18.5 & 18.9 & 21.8 & 21.3 \\
0.96 & 19.0 & 19.2 & 21.2 & 20.9 \\
0.98 & 19.5 & 19.6 & 20.6 & 20.4 \\
1.00 & 20.0 & 20.0 & 20.0 & 20.0 \\
1.02 & 20.5 & 20.4 & 19.5 & 19.6 \\
1.04 & 21.1 & 20.8 & 19.0 & 19.3 \\
1.06 & 21.7 & 21.2 & 18.6 & 18.9 \\
1.08 & 22.3 & 21.7 & 18.1 & 18.6
\end{tabular}

Table 4. Corn consumption, date $2(\beta=1, k=10, \tau=.01)$

\begin{tabular}{ccccc}
\hline$\alpha$ & $x_{1}(E 2 c)$ & $x_{2}(W 2 c)$ & $x_{3}(W 2 c)$ & $x_{4}(E 2 c)$ \\
\hline 0.92 & 18.0 & 21.9 & 22.5 & 18.5 \\
0.94 & 18.5 & 21.3 & 21.8 & 18.9 \\
0.96 & 19.0 & 20.9 & 21.1 & 19.2 \\
0.98 & 19.5 & 20.4 & 20.5 & 19.6 \\
1.00 & 20.0 & 20.0 & 20.0 & 20.0 \\
1.02 & 20.5 & 19.6 & 19.5 & 20.4 \\
1.04 & 21.1 & 19.3 & 19.0 & 20.8 \\
1.06 & 21.7 & 18.9 & 18.6 & 21.3 \\
1.08 & 22.3 & 18.6 & 18.1 & 21.7
\end{tabular}

\section{References}

1. Arnott, RJ and MacKinnon, J (1977) The effects of the property tax: a general equilibrium simulation. Journal of Urban Economics 4:389-407.

2. Berliant, M (1985) Equilibrium models with land: A criticism and an alternative. Regional Science and Urban Economics 15:325-340.

3. Berliant, M and ten Raa, T (1981) On the continuum approach of spatial and some local public good models: Some problems. Journal of Economic Theory 55: $95-120$.

4. Debreu, G (1959) Theory of Value. John Wiley, New York.

5. Fujita, M. Krugman, P and Venables, A (2000). The Spatial Economy. MIT Press, Cambridge, MA.

6. Hart, S, Hildenbrand, W and Kohlberg, E (1974) On equilibrium allocations as distributions on the commodity space. Journal of Mathematical Economics 1:159-166.

7. Krugman, P (1991) Increasing returns and economic geography. Journal of Political Economy 99: 483-499.

8. McKenzie, L (1959) On the existence of general equilibrium for a competitive market. Econometrica 27: 54-71.

9. Papageorgiou, Y and Pines, D (1989) The logical foundations of urban economics are consistent. Journal of Economic Theory 50:37-53. 
10. Richter, DK (1978) Existence and computation of a Tiebout general equilibrium model. Econometrica 46: 779-805.

11. Richter, DK (1978) The computation of urban land use equilibria. Journal of Economic Theory 19: (1978b) 1-27.

12. Scarf, HE (1967) On the computation of equilibrium prices. in Fellner, WK (ed) Ten Economic Essays in the Tradition of Irving Fisher. John Wiley, New York.

13. Sjaastad, LA (1962) The costs and returns of human migration. Journal of Political Economy 70:380-393.

14. Starrett, D (1978) Market allocation of location choice in a model with free mobility. Journal of Economic Theory 17: 21-37. 